\title{
Scattering of Dirac electrons from a skyrmion: Emergence of robust skew scattering
}

\author{
Cheng-Zhen Wang $\odot,{ }^{1}$ Hong-Ya Xu, ${ }^{1}$ and Ying-Cheng Lai $\oplus^{1,2, *}$ \\ ${ }^{1}$ School of Electrical, Computer and Energy Engineering, Arizona State University, Tempe, Arizona 85287, USA \\ ${ }^{2}$ Department of Physics, Arizona State University, Tempe, Arizona 85287, USA
}

(Received 20 November 2019; accepted 7 February 2020; published 3 March 2020)

\begin{abstract}
We study electron scattering from a closed magnetic structure embedded in the top surface of a topological insulator. Outside the structure there is a uniform layer of ferromagnetic insulators, leading to a positive effective mass for the Dirac electrons. The mass inside the structure can be engineered to be negative, leading to a skyrmion structure. The geometric shape of the structure can be circular or deformed, leading to integrable or chaotic dynamics, respectively, in the classical limit. For a circular structure, the relativistic quantum scattering characteristics can be calculated analytically. For a deformed structure, we develop an efficient numerical method, the multiple-multipole method, to solve the scattering wave functions. We find that, for scattering from a skyrmion, an anomalous Hall effect, as characterized by strong skew scattering, can arise, which is robust against structural deformation due to the emergence of resonant modes. In the short- (long-)wavelength regime, the resonant modes manifest themselves as confined vortices (excited edge states). The origin of the resonant states is the spin phase factor of massive Dirac electrons at the skyrmion boundary. Further, in the short-wavelength regime, for a circular skyrmion, a large number of angular momentum channels contribute to the resonant modes. In this regime, in principle, classical dynamics is relevant, but we find that geometric deformations, even those as severe as leading to fully developed chaos, have little effect on the resonant modes. The vortex structure of the resonant states makes it possible to electrically charge the skyrmion, rendering feasible the electrical manipulation of its motion. In the long-wavelength regime, only the lowest angular momentum channels contribute to the resonant modes, making the skew scattering sharply directional. These phenomena can be exploited for applications in generating dynamic skyrmions for information storage and in Hall devices.
\end{abstract}

DOI: 10.1103/PhysRevResearch.2.013247

\section{INTRODUCTION}

This paper is devoted to studying relativistic quantum scattering of Dirac electrons in systems involving magnetism. There are two motivations. First, quantum scattering of spin$1 / 2$ fermions is fundamental to developing two-dimensional (2D) Dirac-material-based devices. Second, magnetic materials have been efficient carriers of information and the physics of magnetic textures has been a topic of significant interest. In general, in quantum scattering, the nature of the underlying classical dynamics can play a role. For example, consider electronic scattering from a $2 \mathrm{D}$ electrical potential domain generated by an external gate voltage. In the classical limit of zero wavelength, the electrons are point particles and the domain is effectively a $2 \mathrm{D}$ billiard system in which electrons move along straight lines and are reflected when hitting the boundary. For a circular domain, the classical dynamics are integrable. However, for a deformed domain, e.g., a stadiumshaped domain, the classical dynamics can be ergodic in the

\footnotetext{
*ying-cheng.lai@asu.edu

Published by the American Physical Society under the terms of the Creative Commons Attribution 4.0 International license. Further distribution of this work must maintain attribution to the author(s) and the published article's title, journal citation, and DOI.
}

phase space. In this case, there is sensitive dependence on initial condition because two nearby trajectories will diverge from each other exponentially, the hallmark of chaos. Since geometric deformations are inevitable in applications, it is necessary in the study of quantum scattering to take into account the nature of classical dynamics. In particular, it is useful to consider deformed domains to uncover the possible effects of classical chaos on quantum scattering.

We employ the setting of a $2 \mathrm{D}$ closed magnetic structure embedded in a uniform layer of ferromagnetic insulator (FMI) materials on the top of a 3D topological insulator (TI). Outside the structure, due to the FMI layer and the proximity effect, the electrons obey the Dirac equation with a positive mass. The mass of the closed structure can be engineered to be negative, making it a skyrmion [1-4]. The skyrmion structure can be deformed so that the classical particle motion inside is chaotic. The massive Dirac electrons moving on the surface of the TI are scattered by the structure. The system thus not only provides a setting for exploring new physics associated with scattering of Dirac electrons from a magnetic skyrmion for applications, e.g., in spintronics, but also represents a paradigm to study the effects of classical chaos on relativistic quantum scattering in the presence of magnetism.

To be systematic and general, we consider the cases where the magnetic structure on top of the TI can be of either the skyrmion or the nonskyrmion type. The structure can simply be a circle, in which case the classical dynamics is 
integrable, or it can be deformed from the circular shape, e.g., a stadium, where there is fully developed chaos in the classical limit. For a circular structure, the various scattering cross sections can be obtained analytically from the standard partial-wave analysis. For a deformed structure, we adopt an efficient method, the multiple multipole (MMP) method in optics, to solving the scattering wave functions of the twocomponent Dirac fermion in the magnetic system. We focus on two regimes: the short-wavelength regime, where the size of the magnetic structure is larger than the wavelength so that the underlying classical dynamics is relevant, and the longwavelength regime, where the structure size is comparable to or smaller than the wavelength. There are two main results. First, a skyrmion can lead to strong skew scattering due to the emergence of resonant modes that manifest themselves as confined vortices inside the skyrmion in the short-wavelength regime or confined edge states in the long-wavelength regime. The resonant modes are the result of mass sign change across the skyrmion boundary. For a circular skyrmion, in the shortwavelength regime, a large number of angular momentum channels contribute to the resonant modes and electron charging arises, providing a way to electrically manipulate the skyrmion motion. In the long-wavelength regime, only the lowest angular momentum channels contribute to the resonant states, leading to strongly directional skew scattering with implications in developing Hall devices. The second result is that classical chaos generated by geometrical deformations has little effect on the scattering from a skyrmion. The scattering phenomena uncovered for the circular case are thus robust. The immunity of the scattering dynamics to severe deformation of the skyrmion structure is advantageous for spintronic device applications.

This paper is organized as follows. In Sec. II we provide the background of our research in terms of magnetic skyrmion, TIs, the marriage between the skyrmion and TI, and relativistic quantum chaos. In Sec. III we describe the Hamiltonian and outline the methods (analytic and numerical). In Sec. IV we demonstrate the emergence of robust resonant states in scattering from the skyrmion for both integrable and chaotic classical dynamics. In Sec. V we develop a partial-wavedecomposition-based analysis for resonances associated with scattering corresponding to classical integrable dynamics. In Sec. VI we summarize the main findings and discuss experimental feasibility and open issues. Finally, in the Appendix we detail the MMP method developed for numerically calculating the scattering wave functions associated with deformed domain hosting chaotic dynamics in the classical limit.

\section{BACKGROUND}

\section{A. Magnetic skyrmion}

Generally, a skyrmion is a particlelike magnetic excitation with a swirling topological 2D spin texture, i.e., the spin at the core and the spin at the perimeter point are in opposite directions [1-4]. The small size of the skyrmions and the possibility of moving them with electrical currents of small density $\left(\sim 10^{5} \mathrm{~A} / \mathrm{m}^{2}\right)$ make them promising candidates for spintronic storage or logic devices [1,2]. Skyrmions have been experimentally observed in chiral magnets $[5,6]$ as a result of the competition between the Dzyaloshiskii-Moriya (DM) interactions, Heisenberg exchange, and Zeeman interactions. It has been demonstrated that metallic skyrmions can be driven by spin transfer torque from the electric current [7-9]. Optical skyrmion lattices have been achieved in an evanescent electromagnetic field [10]. In addition, the topological spin Hall effect has been demonstrated in which a pure transverse spin current is generated from a skyrmion spin texture [11-15].

\section{B. Topological insulators}

Topological insulators are quantum materials with surface states residing in the bulk insulating gap $[16,17]$. The edge states are topologically protected and are robust against nonmagnetic disorders due to a strong spin-momentum locking. The electron motion on the surface follows the 2D linear dispersion with a single band-touching Dirac point and are described by the Dirac equation. In spite of the strong spinmomentum locking, the surface electronic states are sensitive to magnetic perturbations. That is, the electrons will be scattered off upon encountering a magnetic structure on the surface of the TI. The interaction between the topological surface states and magnetic materials in a quasi-onedimensional setting has been studied [18-20] where, due to the spin-momentum locking, the exchange coupling between the magnetization and the surface electronic states can lead to intriguing phenomena such as anomalous magnetoresistance and unconventional transport behaviors [21,22]. The interaction can also lead to nonlinear or even chaotic dynamics in the evolution of magnetization of the FMI [23,24]. For example, complicated dynamics can emerge in the magnetization switching due to a Hall-current-induced effective anisotropic field [18,25-27] and steady self-oscillations can arise in an FMI/TI heterostructure [28-30]. A quite recent computational study has revealed phase locking in the magnetization dynamics of two FMIs on top of a 3D TI [31].

\section{Skyrmion and TI}

Efforts in improving thermal efficiency and better manipulating skyrmions have led to the marriage between the skyrmion and TI, where skyrmions arise on the surface of a TI. Electric charging of magnetic vortices on the surface of a TI was investigated [32] and the confinement state in the skyrmion structure on the surface of a TI was discovered, paving the way to driving skyrmion motion using an applied electric field [33]. Electron skew scattering induced by the skyrmion structure on the TI surface was also studied [34]. Quite recently, the combination of two skyrmions with opposite winding numbers, called skyrmionium, in an FMI/TI heterostructure was observed in the physical space [35-37]. Theoretically, fluctuation-induced Néel and Bloch skyrmions on the surface of a TI have been predicted [38].

Previous studies focused on scattering of electrons from radially symmetric skyrmion structures. Deformed skyrmion structure has been studied in recent years. For example, it was found that Majorana modes are robust against skyrmion deformations [39]. Quantum engineering of Majorana fermions in a deformed skyrmion structure was also studied $[40,41]$ and 
deformed (elongated) skyrmions were used for stabilization and control of Majorana bound states in proximity to an $s$ wave superconductor [42]. Shape-dependent resonant modes have been discovered recently in skyrmions in magnetic nanodisks [43].

\section{Relativistic quantum chaos}

The study of the manifestations of classical chaos in relativistic quantum systems was pioneered by Berry and Mondragon [44] and recently emerged as an interdisciplinary field of research $[45,46]$ with applications to Dirac material systems $[47,48]$. In contrast to the traditional field of (nonrelativistic) quantum chaos $[49,50]$, where classical chaos often bears strong signatures in the corresponding quantum systems, such identifying characteristics tend to be weakened in the relativistic quantum counterparts [51,52]. For example, in scattering, e.g., electronic transport through a quantum-dot structure, chaos tends to smooth out fluctuations in scattering matrix elements, quantum transmission, or conductance [53-58] if the quantum behaviors are governed by the Schrödinger equation. However, in 2D Dirac materials such as graphene, strong fluctuations of the quantum scattering characteristics can persist to a certain extent in spite of classical chaos $[59,60]$. Another example is a 2D deformed ring with a line of magnetic flux through the center, where Schrödinger electrons are localized but Dirac electrons can keep circulating along the edges of the ring domain, generating a superpersistent current in spite of fully developed classical chaos in the domain [61], a phenomenon that can be exploited for creating a robust relativistic qubit [62]. Quite recently, the weakening of the manifestations of chaos in spin-1/2 Dirac fermion systems was studied [52] using the approach of an out-of-time-ordered correlator [63]. It has also been revealed that, for scattering in spin-1 Dirac-Weyl fermion systems, a class of robust resonant modes can emerge that defy classical chaos completely [51].

\section{MODEL AND METHOD}

We place an FMI thin film, e.g., $\mathrm{Cu}_{2} \mathrm{OSeO}_{3}$, on top of a TI with a single magnetic structure at the center of the thin film, as schematically illustrated in Fig. 1. The motion of the surface electrons is affected by the structure with the magnetization vector $\boldsymbol{n}(\boldsymbol{r})$. The Hamiltonian of the system is

$$
H=v_{F}(\hat{\boldsymbol{p}} \times \boldsymbol{\sigma})_{z}-\Delta_{s} \boldsymbol{n}(\boldsymbol{r}) \cdot \boldsymbol{\sigma},
$$

where $v_{F}$ is the Fermi velocity, $\hat{\boldsymbol{p}}=-i \nabla$ is the momentum operator, $\sigma=\left(\sigma_{x}, \sigma_{y}, \sigma_{z}\right)$ are the Pauli matrices, and $\Delta_{s}(>0)$ is the spin-splitting energy from the exchange interaction between the electron and the magnetization. In polar coordinates $\boldsymbol{r}=(r, \theta)$, for a circular structure, the magnetization vector can be parametrized as

$$
\boldsymbol{n}(\boldsymbol{r})=\left[-\sin \theta \sqrt{1-n_{z}^{2}(r)}, \cos \theta \sqrt{1-n_{z}^{2}(r)}, n_{z}(r)\right] .
$$

For a deformed magnetic structure, there is swirling spin texture with magnetic moment points up on the edge and down in the center [64]. The out-of-plane component of the magnetic texture $n_{z}(\boldsymbol{r})$ acts as a Dirac mass term, which opens a gap in the electronic band structure. The in-plane component (a)

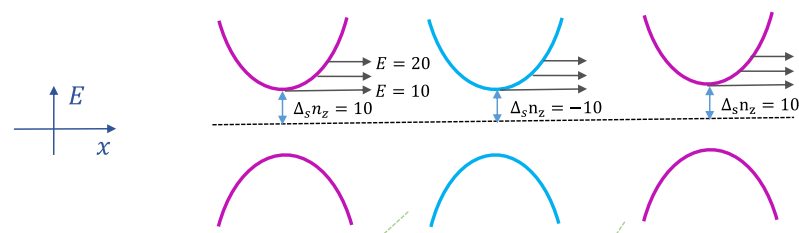

(b)

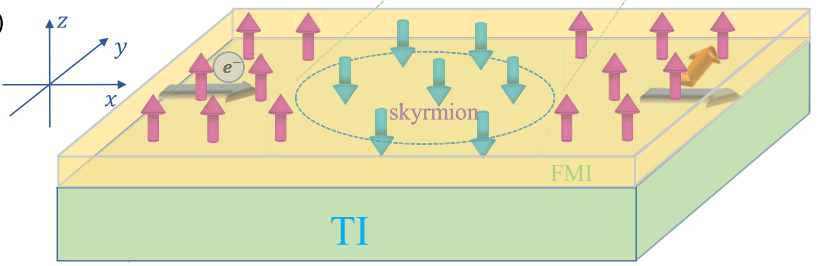

FIG. 1. Schematic illustration of electron scattering from a skyrmion structure in a thin FMI film deposited on top of a TI. (a) Band structure of the FMI/TI heterostructure. Outside (inside) the skyrmion structure, the mass corresponding to the band gap is positive (negative). (b) Illustration of electron scattering behavior from the skyrmion structure. The red (up) and blue (down) arrows represent the magnetization vector $\boldsymbol{n}(\boldsymbol{r})$. For electronic states outside and inside the skyrmion, the associated spin direction is different due to the opposite signs of mass.

$\boldsymbol{n}_{\|}$can lead to an emergent magnetic field in the form

$$
\boldsymbol{B}(\boldsymbol{r})=\frac{c \Delta \operatorname{div}_{\|}(\boldsymbol{r})}{e \hbar v_{F}} .
$$

For a swirling skyrmion structure, the emergent magnetic field $\boldsymbol{B}$ is zero and the in-plane component can be gauged away $[33,34]$. In this case, the hard-wall approximation $n_{z}(\boldsymbol{r})= \pm 1$ can be invoked $[33,34]$, with the points inside and outside the skyrmion structure taking on the value of minus one and one $\left(n_{1}=1\right.$ and $\left.n_{2}=-1\right)$, respectively. In experiments, such a structure can be realized using materials with a strong out-of-plane magnetic anisotropy. In our study, we assume that the magnetic structure is fixed and unaffected by the interface electrons. Experimentally, a skyrmion structure can be stabilized via the DM interaction in the FMI [1,2], where the skyrmion size depends on materials parameters such as the relative strength of the Heisenberg and DM exchange interactions [1,2]. Our model is valid for skyrmions with a vortical magnetic texture as described. However, for hedgehog skyrmions, the in-plane magnetic field cannot be gauged away due to the emergent magnetic flux and the structure is not as stable as vortical skyrmions [1,2].

The energy-momentum dispersion for electrons in free space with a uniform magnetic texture (constant mass) is given by

$$
E_{ \pm}= \pm \sqrt{\hbar^{2} v_{F}^{2}\left(k_{x}^{2}+k_{y}^{2}\right)+\Delta^{2} n_{z}^{2}}
$$

as shown in Fig. 1(a). While the energy dispersion curve inside the skyrmion appears similar to that outside the skyrmion, the spin direction is different for the electronic state due to the opposite signs of mass. An electron will then go through a scattering process in this 2D system. Because of the breaking of the time-reversal symmetry, skew scattering will arise.

For a circular magnetic structure, the scattering wave function and the related behavior can be solved analytically 
using the partial-wave-decomposition method (Sec. V). For a deformed skyrmion, analytic solutions of the scattering wave function are not feasible. We have developed an MMP-based method, which has its origin in optics [65-69] and has recently been extended to scattering of pseudospin-1 particles [51]. The basic idea is to assume two sets of fictitious poles along and in the vicinity of the entire boundary of the magnetic structure: one outside and another inside the boundary. Each pole emits a wave in the form of a Hankel function (spherical wave in the far field). The transmitted wave function at each point inside the scatterer can be expressed as the superposition of the waves emitted by the poles outside the scatterer. Similarly, the refracted wave function at each point outside the scatterer can be written as the combination of the waves emitted by the poles inside the scatterer. The incident plane wave and the reflected and transmitted waves are matched on the boundary to enable the poles to be determined, and the expansion coefficients can be obtained by solving the matrix eigenfunctions. (The details of the MMP method adopted for scattering from a magnetic structure are given in the Appendix.) We validate the method by comparing the MMP solutions with the analytic solution based on partial-wave expansion for a circular skyrmion. Overall, the MMP method is effective and efficient for solving both the near- and far-field scattering problem for a magnetic scatterer of arbitrary shape.

In our calculation, we use the dimensionless quantity obtained via consideration of the scales of the physical quantities involved. In particular, the energy scale in the FMI/TI heterostructure is on the order of meV. In free space with zero mass, the wave vector corresponding to the energy of $1 \mathrm{meV}$ is $k \sim 1 \mathrm{meV} / \hbar v_{F}=3.04 \times 10^{-3} \mathrm{~nm}^{-1}$. We take the dimensionless radius of the magnetic structure (circular shape) to be $R=1$, which corresponds to a real structure of size of $100 \mathrm{~nm}$. We then set the dimensionless energy corresponding to $1 \mathrm{meV}$ to be $k R=0.304$. For $\Delta=10$, the corresponding energy gap is $10 / 0.304 \approx 33 \mathrm{meV}$.

\section{EMERGENCE OF ROBUST RESONANT STATES IN SCATTERING FROM THE SKYRMION}

\section{A. Short-wavelength regime: Resonant vortices and edge modes}

We concentrate on a regime where the wavelength of the incoming Dirac electron is smaller than the size of the magnetic structure so that the classical dynamics inside the structure is relevant. We consider a circular structure as well as a deformed structure that leads to chaos in the classical limit to identify any effect of chaos on the electron scattering behavior.

\section{Far-field behavior}

Far away from the scattering center, for unit incident density the spinor wave function can be written as

$$
\begin{aligned}
\Psi_{I} & =\Psi_{\mathrm{inc}}+\Psi_{\mathrm{ref}} \\
& \approx C\left(\begin{array}{c}
1 \\
i \frac{\hbar v_{F} k}{E-m_{1}}
\end{array}\right) e^{i k r \cos \theta}+C\left(\begin{array}{c}
e^{-i \theta} \\
i \frac{\hbar v_{F} k}{E-m_{1}}
\end{array}\right) \frac{f(\theta)}{\sqrt{r}} e^{i k r},
\end{aligned}
$$

where $C$ is the normalization factor, $k=\sqrt{k_{x}^{2}+k_{y}^{2}}$ is the electron wave vector, $m_{1}=\Delta_{s} n$ and $m_{2}=\Delta_{s} n^{\prime}$ are the mass
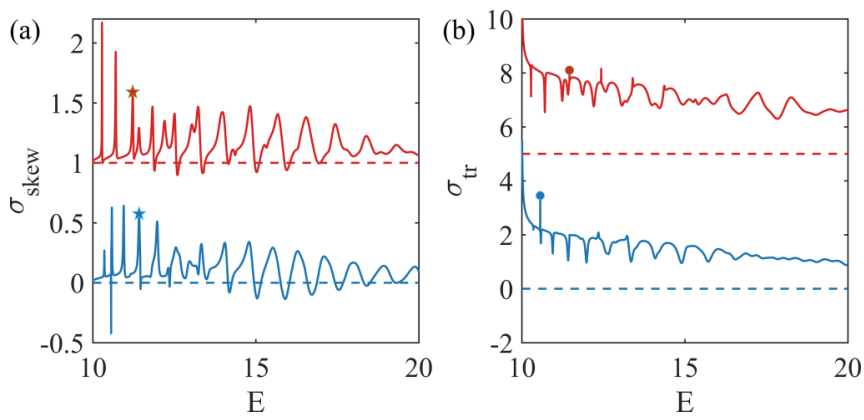

FIG. 2. Skew scattering and transport cross sections versus incident electron energy in the short-wavelength regime. (a) Skew scattering cross section versus the energy. The red and blue curves correspond to a circular and a stadium-shaped skyrmion, respectively. The mass values are $m_{1}=10$ and $m_{2}=-10$. (b) Backscattering cross section as a function of electron energy for the two skyrmion shapes as in (a). In each panel, the red curve has been shifted upward by an amount specified by the horizontal red dashed line for better visualization and comparison with the blue curve.

terms outside and inside the magnetic structure, and $f(\theta)$ denotes the 2D far-field scattering amplitude in the direction defined by the angle $\theta$ with the $x$ axis. For a circular structure, $f(\theta)$ can be obtained analytically. For a chaotic structure, once the reflection function is calculated from the MMP method, $f(\theta)$ can be obtained. The differential cross section is

$$
\frac{d \sigma}{d \theta}=|f(\theta)|^{2} \text {. }
$$

The transport and skew cross sections are defined, respectively, as

$$
\sigma_{t r}=\int_{0}^{2 \pi} d \theta|f(\theta)|^{2}(1-\cos \theta)
$$

and

$$
\sigma_{\text {skew }}=\int_{0}^{2 \pi} d \theta|f(\theta)|^{2} \sin \theta .
$$

Figures 2(a) and 2(b) show, respectively, the skew scattering and transport cross sections as a function of incident electron energy, for a skyrmion (negative value of $m_{2}$ ) of circular shape (upper panel) and stadium shape (lower panel) of the same area $\pi$ in dimensionless units. The stadium shape is chosen because of its mirror symmetry for the incident plane waves so as to avoid an unnecessary complication: mixing of skew scattering and backscattering (or reflection). The aspect ratio for the stadium shape is set to be 2. For both skyrmion shapes, there are sharp resonant peaks in the skew cross section in the lower-energy range close to the gap, an indication of the emergence of the anomalous Hall effect associated with Dirac electron scattering from the skyrmion. As the incident energy is increased, the peak height is reduced but its width becomes larger, as a higher-energy value corresponds to less distortion in the energy-momentum dispersion with the mass gap. Note that there is little difference in the skew scattering crosssection curves for the two skyrmion shapes, indicating that the nature of the classical dynamics hardly affects the scattering. For the curves of the transport cross section, as shown in Fig. 2(b), its value decreases with increasing energy. For 

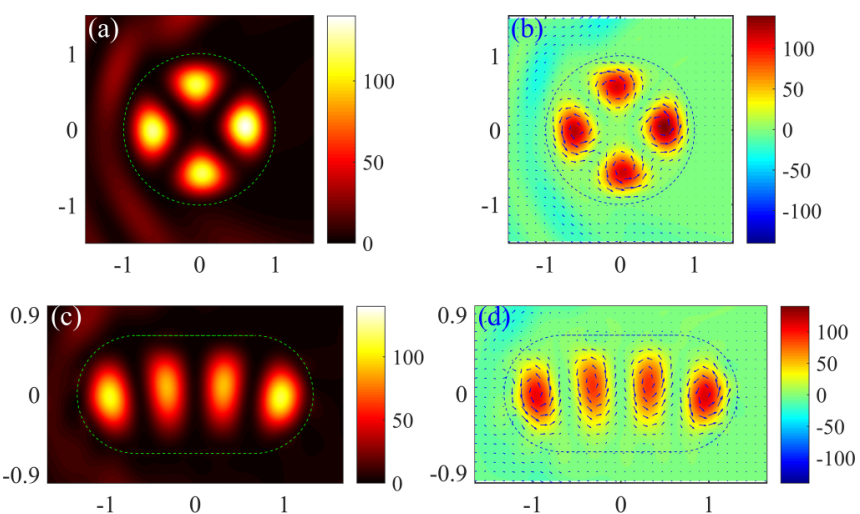

FIG. 3. Probability and current density distribution for selected vortex states. (a) Probability distribution for scattering from a circular skyrmion for $m_{1}=10, m_{2}=-10$, and $E=11.225$. (b) In-plane current (marked by arrows) and spin- $z$ component (color coded) density distribution in the circular skyrmion region. (c) and (d) Corresponding probability, current, and spin distribution for scattering from a stadium-shaped skyrmion for $m_{1}=10, m_{2}=-10$, and $E=$ 11.42 .

low-energy values, the valleys in the transport cross section correspond exactly to the skew scattering peaks. Sharp peaks also exist in the backscattering cross-section curve. Similar to the skew cross section, the nature of the classical dynamics has no appreciable effect. The results in Fig. 2 indicate that skyrmion skew scattering is robust against geometric deformations that are so severe as to change the classical behavior completely: from integrable dynamics to chaos.

\section{Near-field behavior}

To understand the origin of the deformation- (chaos-) independent far-field scattering (transport) behavior, we study the near-field scattering behavior by examining the probability density and the current density distribution associated with some specific energy state. In particular, the probability density is given by $P=\Psi^{\dagger} \Psi$, where $\Psi=\left(\psi_{1}, \psi_{2}\right)^{T}$ is the wave function, and the probability current operator is $\hat{J}=$ $\nabla_{p} H=v_{F}\left(\sigma_{y},-\sigma_{x}\right)$. The current density can be obtained as

$$
\boldsymbol{J}=\left(J_{x}, J_{y}\right)=v_{F}\left[2\left(i \psi_{1} \psi_{2}^{*}\right),-2\left(\psi_{1} \psi_{2}^{*}\right)\right] .
$$

The probability density distribution of the spin- $z$ component is given by

$$
\left\langle\sigma_{z}\right\rangle=\left|\psi_{1}\right|^{2}-\left|\psi_{2}\right|^{2} .
$$

We choose a representative energy value corresponding to a skew scattering cross section peak: $E=11.225$ for the circular skyrmion and $E=11.42$ for the stadium-shaped skyrmion, marked as the red and blue stars, respectively, in Fig. 2(a). The probability and the current density distributions are shown in Fig. 3. From both skyrmion structures, there are scattering resonant states, as shown in Figs. 3(a) and 3(c). The resonant patterns correspond to weak backscattering but stronger skew scattering cross sections, indicating that these are effectively quasiconfined states. Further insights into the contribution of the resonant states to skew scattering can be gained by examining the current density distribution (marked by arrows) and the spin- $z$ component density distribution
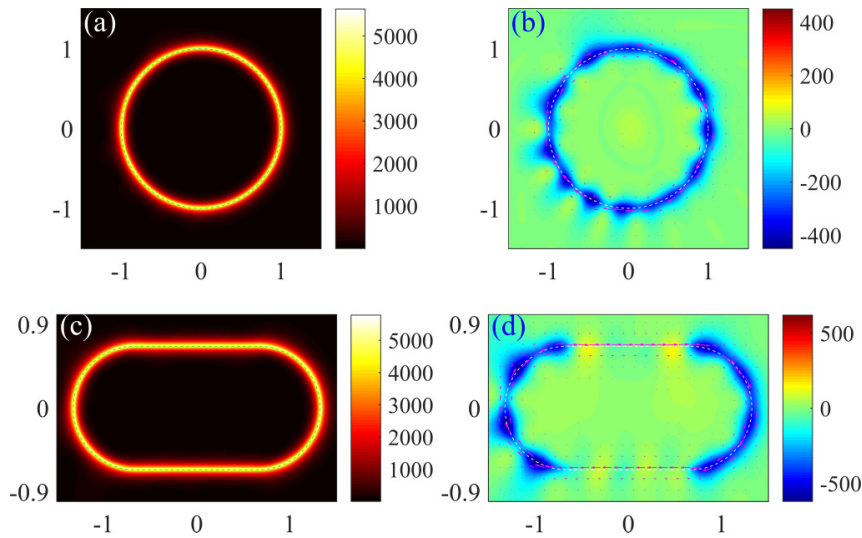

FIG. 4. Wave-function probability and current density distribution associated with selected edge states. (a) Probability distribution for scattering from a circular skyrmion for $m_{1}=10, m_{2}=-10$, and $E=11.461$. (b) Corresponding in-plane current (marked by arrows) and spin- $z$ component (represented by colors) density distribution. (c) and (d) Probability and spin distributions associated with scattering from a stadium-shaped skyrmion for $m_{1}=10, m_{2}=-10$, and $E=10.564$.

(color coded) in the 2D skyrmion structure, as shown in Figs. 3(b) and 3(d). We see that the confined resonant states form vortices with counterclockwise currents. There is also an out-of-plane spin component along the positive $z$ direction. The vortices have an apparent directionality, so they can affect the skew scattering direction and magnitude. The vortices are formed by the interference of waves reflected from the boundary and are robust against boundary deformation. As a result, the nature of the classical dynamics, integrable or chaotic, has no significant effect on scattering.

In addition to the confined vortex states inside the skyrmion structure, another form of confined states arises along the skyrmion boundary, as shown in Figs. 4(a) and 4(c), for scattering from a circular and a stadium-shaped skyrmion, respectively. There is strong confinement of the scattering wave function near the boundary with clockwise current and a spin- $z$ component along the negative $z$ axis direction, as shown in Figs. 4(b) and 4(d). The edge states correspond to sharp resonant peaks in the backscattering cross section marked by closed circles in Fig. 2(b). For the circular skyrmion, the edge states have no corresponding sharp peaks in skew scattering. For the stadium-shaped skyrmion, the edges states correspond to sharp valleys in the skew scattering cross section.

\section{B. Long-wavelength regime: Resonant modes near the boundary}

\section{Far-field behavior}

We consider the regime where the skyrmion size is smaller than the electronic wavelength: $R \ll 1 / k$. This can be realized by setting the area of the skyrmion structure to be $0.01 \pi$ for both circular $(R=0.1)$ and stadium-shaped skyrmions. In this long-wavelength regime, for a deformed skyrmion structure, the MMP method is still effective for calculating the far-field cross sections and the near-field state distribution. Representative results on the skew scattering and 



FIG. 5. Characteristics of Dirac electron scattering from a magnetic skyrmion in the long-wavelength regime. (a) Skew scattering and (b) backscattering cross sections versus energy. The red and blue curves correspond to a circular and a stadium-shaped skyrmion, respectively. The mass values are $m_{1}=10$ and $m_{2}=-10$. In each panel, the red curve has been shifted upward for a proper amount for better visualization and comparison with the blue curve.

transport cross sections versus the incident energy are shown in Fig. 5. Different from the scattering behaviors in the shortwavelength regime, the oscillations of the skew scattering cross section with energy are weak. For example, in the energy range $10<E<20$, only one smooth peak appears. There is hardly any difference in the scattering characteristics between the two skyrmion structures, which is understandable as any structural differences are not resolved in the long-wavelength regime. Because of a lack of appreciable oscillations, there is directional skew scattering over a large energy range, a desired feature in Hall device applications.

\section{Near-field behavior}

We examine the state associated with the energy value that leads to the lowest skew scattering cross section: $E=$ 12.072 for the circular and $E=11.46$ for the stadium-shaped skyrmion; the respective probability density distributions are shown in Figs. 6(a) and 6(c). The states are concentrated in the vicinity of the boundary, which are different from the vortex states observed in the short-wavelength regime. The edge states thus represent a different type of resonant states with directional current, as shown in Figs. 6(b) and 6(d). It can be seen that the current direction is downward at the edge, contributing to skew scattering. The spin- $z$ component is along the negative $z$ direction.

\section{Further demonstration of strong skew scattering from a skyrmion structure}

To further demonstrate the shape-independent skew scattering behavior of Dirac electrons from a magnetic structure, we study the effects of changing the mass of the skyrmion texture. To be concrete, we set $m_{1}>0$ and choose a set of positive and negative $m_{2}$ values. In this setting, there is a skyrmion for $m_{2}<0$ but the magnetic structure is a nonskyrmion for $m_{2}>0$.

We first examine the short-wavelength regime to probe into the origin of the emerged confined vortex states. Figures 7(a) and 7(b) show the skew scattering cross sections for the circular and stadium-shaped magnetic structures, respectively, for $m_{1}=10$ and $m_{2}=-9,-5,0,5,9$. It can be seen that,
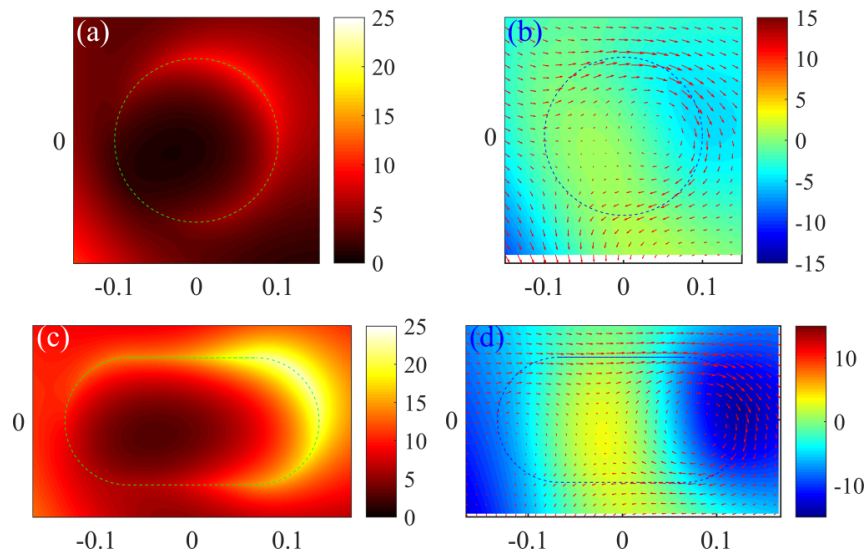

FIG. 6. Wave-function probability and current density distributions for selected states for scattering in the long-wavelength regime. (a) Probability distribution and (b) in-plane current together with the spin- $z$ component density distributions, for scattering from a circular skyrmion for $m_{1}=10, m_{2}=-10$, and $E=12.072$. (c) and (d) Corresponding results for scattering from a stadium-shaped skyrmion for $m_{1}=10, m_{2}=-10$, and $E=11.46$.

among the five cases, the resonant oscillations of the cross section with energy last longer for $m_{2}=-9$. On the contrary, for $m_{2}=9$ (nonskyrmion), the oscillations diminish rapidly as the energy is increased. These behaviors hold regardless of whether the underlying classical dynamics is integrable or chaotic. Overall, a large difference between the masses inside and outside the magnetic structure can lead to stronger and long-lasting resonant modes and consequently to more pronounced skew scattering. Figures 8 (a) and 8 (b) show the probability density distribution for $m_{2}=9$ and $m_{2}=-9$,


FIG. 7. Effects of varying mass on Dirac electron scattering in the short-wavelength regime. The area of the magnetic structure is $\pi$. (a) Skew scattering cross section versus the electron energy for a circular structure for mass values $m_{2}=-9,-5,0,5,9$, represented by the red, orange, green, blue, and purple solid curves, respectively. In each panel, the curves have been shifted upward for better visualization and comparison, where each horizontal dashed line denotes the zero reference point. The mass outside the magnetic structure is $m_{1}=10$. (b) Corresponding curves for a stadium-shape structure with the same mass values as in (a). 

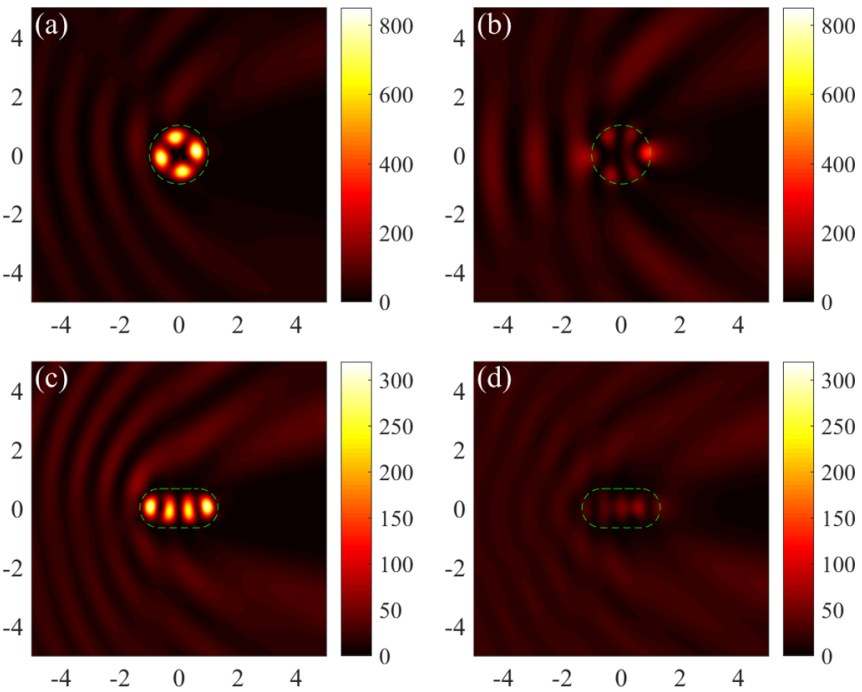

FIG. 8. Probability density distribution for selected states in the circular and stadium-shaped structures for different masses in the short-wavelength regime. (a) Circular skyrmion structure $\left(m_{2}=-9\right)$ for $E=10.349$, (b) circular nonskyrmion structure $\left(m_{2}=9\right)$ for $E=$ 10.234 , (c) stadium-shaped skyrmion $\left(m_{2}=-9\right)$ for $E=10.552$, and (d) stadium-shaped structure $\left(m_{2}=9\right)$ for $E=10.514$.

respectively, for the circular magnetic structure. The corresponding results for the stadium-shaped structure are shown in Figs. 8(c) and 8(d). For both structures, there are resonant modes for $m_{2}=-9$ (when the magnetic structure is of the skyrmion type) but not for the case of $m_{2}=9$.

In the long-wavelength regime, regardless of the shape of the magnetic structure (circular or stadium shaped), the skew scattering cross section decreases as the relative mass difference is reduced, as shown in Fig. 9 for $m_{2}=-9,-5,0,5,9$. Figure 10 shows representative resonant states for the circular and stadium-shaped structures for $m_{2}= \pm 9$. Again, when the magnetic structure is of the skyrmion type, skew scattering is strong, making the scattering electrons directional. However, when the structure is not of the skyrmion type, skew scattering is weak.

\section{PARTIAL-WAVE-DECOMPOSITION-BASED ANALYSIS}

Numerically, we have observed strong skew scattering of Dirac electrons from a skyrmion structure, which is robust against geometric deformation. We now provide an analytic understanding of skew scattering based on the method of partial-wave decomposition. Consider a circular skyrmion. Key to pronounced skew scattering is the resonant modes emerged from the scattering process. In the short-wavelength regime, a large number of angular momentum components are involved in the scattering, leading to a large number of resonant modes as the result of various combinations of the angular momentum components, which are manifested as peaks in the curve of the cross section with the energy. In the long-wavelength regime, typically only a single resonant mode is dominant, implying the involvement of only the several lowest angular momentum components. The asymmetric contribution from different angular momentum channels leads to the observed pronounced skew scattering.
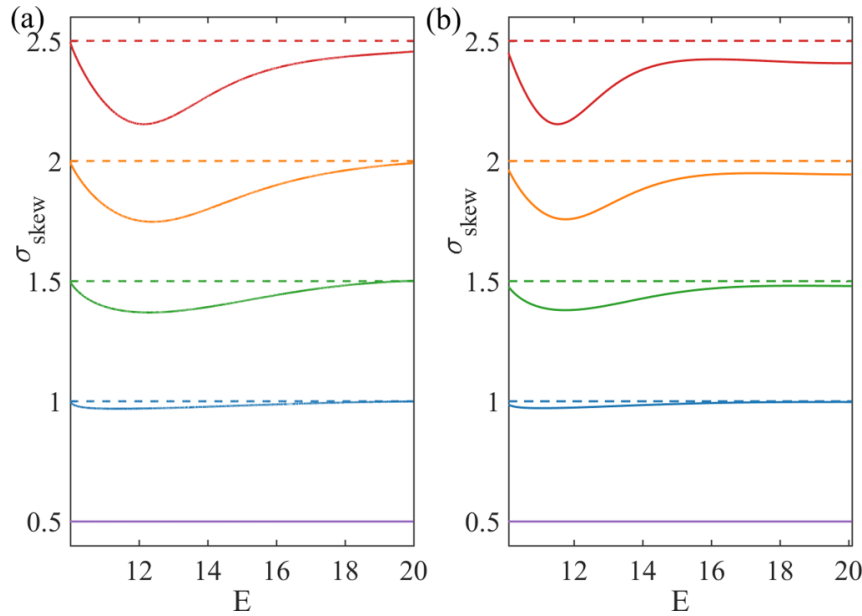

FIG. 9. Skew scattering for different mass values of the magnetic structure in the long-wavelength regime. The area of the structure is $\pi / 100$ and the mass outside the structure is $m_{1}=10$. (a) For a circular structure, skew scattering cross section for $m_{2}=-9,-5,0,5,9$, represented by the red, orange, green, blue, and purple solid curves, respectively. In each panel, the curves have been shifted upward for better visualization and comparison, with the horizontal dashed lines denoting the zero reference point. (b) Corresponding results for a stadium-shaped magnetic structure.

Because the circular and stadium-shaped skyrmion structures generate similar scattering behavior, the analytic results from the circular skyrmion case also provides an understanding of the emergence of strong skew scattering in the stadium-shaped skyrmion.

For a circular skyrmion, the rotational symmetry stipulates conservation of the total angular momentum $\hat{J}_{z},\left[\hat{J}_{z}, H\right]=0$,
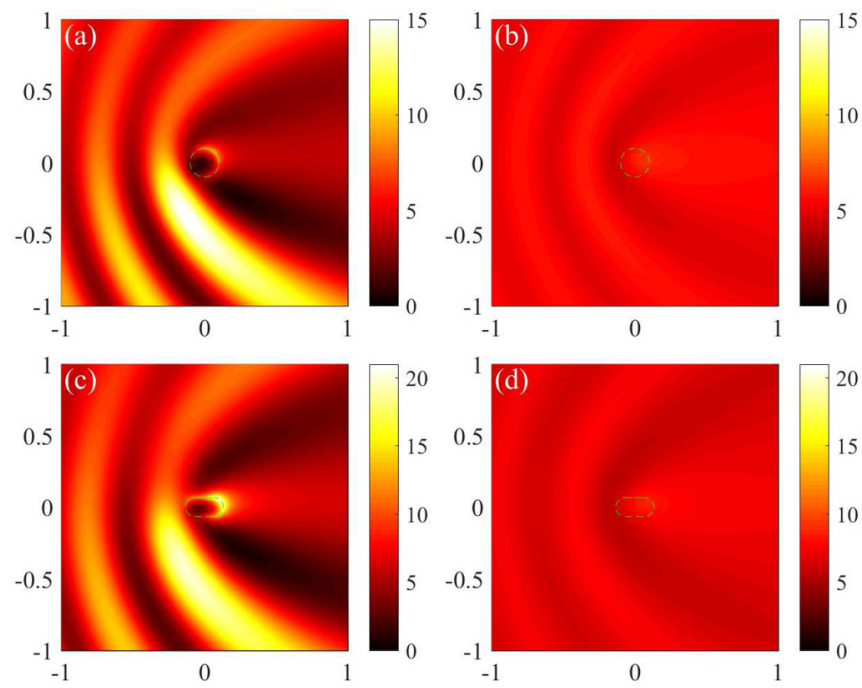

FIG. 10. Probability density distribution for the states corresponding to the minimum of the skew scattering cross section in circular and stadium-shaped magnetic structures in the longwavelength regime: (a) a circular skyrmion structure for $m_{2}=-9$ and $E=12.152$, (b) a circular nonskyrmion structure for $m_{2}=9$ and $E=12.317$, (c) a stadium-shaped skyrmion structure for $m_{2}=-9$ and $E=11.53$, and (d) a stadium-shaped nonskyrmion structure for $m_{2}=9$ and $E=11.72$. 
and the partial-wave component with total angular momentum $j(= \pm 1 / 2, \pm 3 / 2, \ldots)$ in polar coordinates $(r, \theta)$ can be written as

$$
\psi_{j}(\boldsymbol{r})=\left(\begin{array}{l}
u_{j}(r) e^{i(j-1 / 2) \theta} \\
v_{j}(r) e^{i(j+1 / 2) \theta}
\end{array}\right) .
$$

The Hamiltonian in the polar coordinates is

$$
H=\hbar v_{F}\left(\begin{array}{cc}
-\frac{\Delta_{s} n}{\hbar v_{F}} & -e^{-i \theta} \frac{\partial}{\partial r}+e^{-i \theta} \frac{i \partial}{r \partial \theta} \\
e^{i \theta} \frac{\partial}{\partial r}+e^{i \theta} \frac{i \partial}{r \partial \theta} & \frac{\Delta_{s} n}{\hbar v_{F}}
\end{array}\right) .
$$

Substituting the partial-wave form from Eq. (9) into the Hamiltonian (10) leads to an eigenvalue problem and consequently to the explicit expression for the partial waves.

The transmitted wave inside the skyrmion structure $(r<R)$ can be expanded in terms of the partial waves as

$$
\psi^{T}(r, \theta)=C \sum_{l=-\infty}^{\infty} i^{l-1} B_{l}\left(\begin{array}{c}
J_{l-1}\left(k^{\prime} r\right) e^{i(l-1) \theta} \\
-\frac{\hbar v_{F} k^{\prime}}{E-\Delta_{s} n^{\prime}} J_{l}\left(k^{\prime} r\right) e^{i l \theta}
\end{array}\right)
$$

and the reflected wave outside the skyrmion $(r>R)$ can be written as

$$
\psi^{R}(r, \theta)=C \sum_{l=-\infty}^{\infty} i^{l-1} A_{l}\left(\begin{array}{c}
H_{l-1}(k r) e^{i(l-1) \theta} \\
-\frac{\hbar v_{F} k}{E-\Delta_{s} n} J_{l}(k r) e^{i l \theta}
\end{array}\right),
$$

where $C$ is a normalization factor and $l=j+1=2, A_{l}$ and $B_{l}$ are the partial-wave expansion coefficients, $J_{l}$ is the Bessel function of the first kind, and $H_{l}$ is the Hankel function of the first kind of integer order $l$. We denote by $m_{1}=\Delta_{s} n\left(m_{2}=\right.$ $\left.\Delta_{s} n^{\prime}\right)$ the mass term and by $k=\sqrt{\frac{E^{2}-\Delta_{s}^{2} n^{2}}{\hbar^{2} v_{F}^{2}}}\left(k^{\prime}=\sqrt{\frac{E^{2}-\Delta_{s}^{2} n^{2}}{\hbar^{2} v_{F}^{2}}}\right)$ the wave vector outside (inside) the skyrmion structure. For the incident electron in the free region outside the skyrmion structure, the wave function is

$$
\psi^{I}=C\left(\begin{array}{c}
1 \\
i \frac{\hbar v_{F} k}{E-\Delta_{s} n}
\end{array}\right) e^{i k r \cos \theta} .
$$

Using the Jacobi-Anger identity

$$
e^{i z \cos \theta} \equiv \sum_{l=-\infty}^{\infty} i^{l} J_{l}(z) e^{i l \theta},
$$

we can expand the plane wave in the form

$$
\psi^{I}=C \sum_{l} i^{l-1}\left(\begin{array}{c}
J_{l-1} e^{i(l-1) \theta} \\
-\frac{\hbar v_{F} k}{E-\Delta_{s} n} J_{l}(k r) e^{i l \theta}
\end{array}\right) .
$$

Matching the waves at the skyrmion boundary $(r=R)$

$$
\psi^{I}(R)+\psi^{R}(R)=\psi^{T}(R),
$$

we get, after some algebraic manipulation,

$$
A_{l}=\frac{J_{l-1}(k R) J_{l}\left(k^{\prime} R\right)-\frac{\tau}{\tau^{\prime}} J_{l}(k R) J_{l-1}\left(k^{\prime} R\right)}{\frac{\tau}{\tau^{\prime}} H_{l}(k R) J_{l-1}\left(k^{\prime} R\right)-H_{l-1}(k R) J_{l}\left(k^{\prime} R\right)}
$$

and

$$
B_{l}=\frac{J_{l-1}(k R) H_{l}(k R)-J_{l}(k R) H_{l-1}(k R)}{H_{l}(k R) J_{l-1}\left(k^{\prime} R\right)-\frac{\tau^{\prime}}{\tau} H_{l-1}(k R) J_{l}\left(k^{\prime} R\right)},
$$
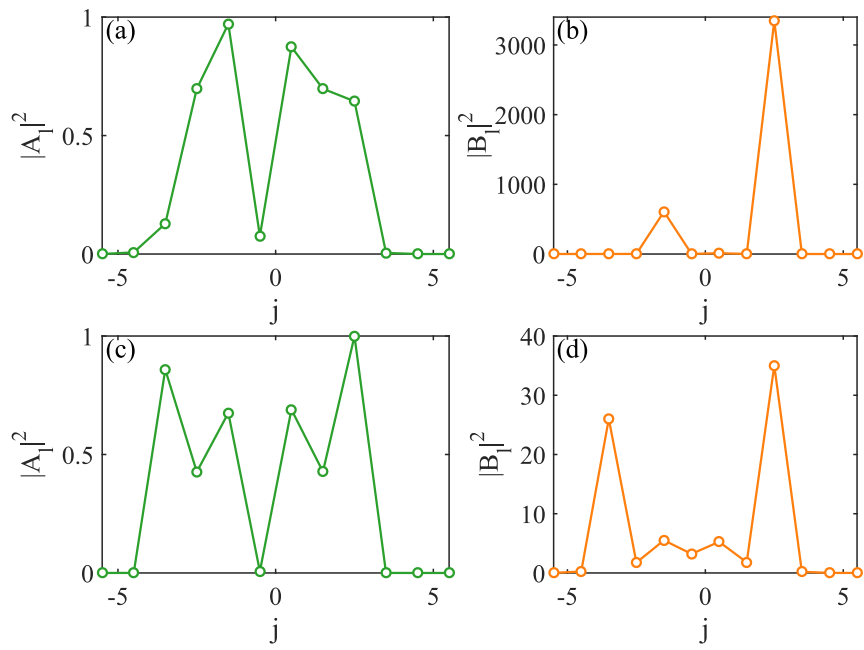

FIG. 11. Partial-wave-decomposition coefficients as a function of total angular momentum for a circular magnetic structure in the short-wavelength regime. Among the quantities plotted, the $A_{l}$ 's are the coefficients for the reflected waves outside the structure and the $B_{l}$ 's are the transmitted wave coefficients. For a skyrmion structure $\left(m_{1}=10\right.$ and $\left.m_{2}=-9\right)$, (a) $\left|A_{l}\right|^{2}$ and (b) $\left|B_{l}\right|^{2}$ are plotted as a function of $j$, where the corresponding state is shown in Fig. 8(a). For a nonskyrmion structure $\left(m_{1}=10\right.$ and $\left.m_{2}=9\right)$, (c) $\left|A_{l}\right|^{2}$ and (d) $\left|B_{l}\right|^{2}$ are plotted versus $j$, where the corresponding state is shown in Fig. 8(b).

where

$$
\tau=-\frac{\hbar v_{F} k}{E-\Delta_{s} n}, \quad \tau^{\prime}=-\frac{\hbar v_{F} k^{\prime}}{E-\Delta_{s} n^{\prime}} .
$$

Using the explicit formulas for $A_{l}$ and $B_{l}$ as given in Eqs. (17) and (18), respectively, we obtain the decomposition coefficients versus the total angular momentum for $R=1$. Figures 11(a) and 11(b) show, for the case of scattering from a skyrmion structure $\left(m_{1}=10\right.$ and $\left.m_{2}=-9\right)$, the expansion coefficients versus the total angular momentum $j$. Figures 11(c) and 11(d) show the corresponding results for the nonskyrmion case $\left(m_{1}=10\right.$ and $\left.m_{2}=9\right)$. It can be seen that several angular momentum components contribute to the reflected wave component $A_{l}$ and the asymmetric distribution of the angular momentum components about zero leads to skew scattering. For the transmitted wave components, the distribution of the angular components is asymmetric as well, leading to the emergence of resonant vortices. For the $B_{l}$ coefficients, their values for the nonskyrmion case are much smaller than those for the skyrmion case, indicating that the skyrmion structure can confine the electrons much more effectively than the nonskyrmion structure.

Setting $R=0.1$ lands the scattering system in the longwavelength regime. Figures 12(a) and 12(b) and Figs. 12(c) and 12(d) show the coefficients associated with different angular momentum components for the skyrmion $\left(m_{1}=10\right.$ and $\left.m_{2}=-9\right)$ and nonskyrmion $\left(m_{1}=10\right.$ and $\left.m_{2}=9\right)$ cases, respectively. In both cases, only a single angular momentum component contributes to the coefficient $A_{l}$, i.e., $j=-1 / 2$, giving rise to the directionality in the scattering and a slow change in the resonant cross section with the energy. The value of $A_{l}$ for the nonskyrmion case is much smaller than that of the 

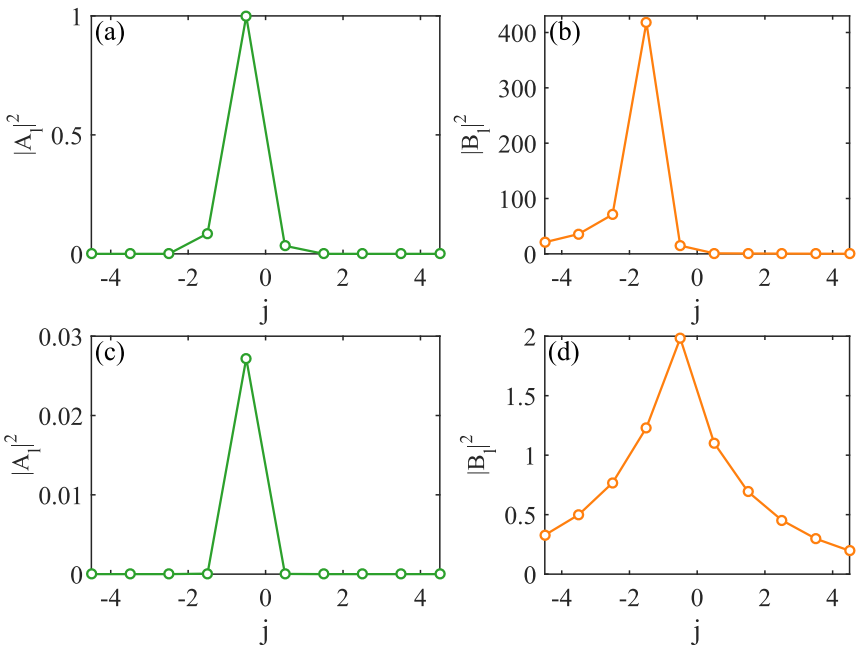

FIG. 12. Transmitted and reflected partial-wave coefficients as a function of the total angular momentum for a circular magnetic structure in the long-wavelength regime. The radius of the structure is $R=0.1$. (a) $\left|A_{l}\right|^{2}$ and (b) $\left|B_{l}\right|^{2}$ are plotted versus $j$ for $m_{1}=10$ and $m_{2}=-9$ (skyrmion case), respectively, where the state is the one shown in Fig. 10(a). (c) $\left|A_{l}\right|^{2}$ and (d) $\left|B_{l}\right|^{2}$ are plotted versus $j$ for $m_{1}=10$ and $m_{2}=9$ (nonskyrmion case), respectively, where the corresponding state is shown in Fig. 10(b).

skyrmion case. For the transmitted coefficient $B_{l}$, the angular momentum component $j=-3 / 2$ dominates the skyrmion case and a number of components including $j=-1 / 2$ have contributions in the nonskyrmion case, and the values of $B_{l}$ are much larger in the skyrmion case than in the nonskyrmion case, again implying stronger confinement by resonance and better directionality of scattering in the skyrmion structure as compared with those in the nonskyrmion structure.

\section{DISCUSSION}

We have investigated relativistic quantum scattering of Dirac electrons from a closed magnetic structure embedded in the top surface of a 3D TI. Outside the structure, there is a uniform FMI layer, leading to a finite but positive mass for the Dirac electron. The mass of the structure itself can be engineered to be negative or positive, where a skyrmion and a nonskyrmion structure arise in the former and latter cases, respectively. In the short-wavelength regime, the nature of the classical dynamics in the closed structure should be relevant to the quantum scattering dynamics, according to conventional wisdom from the study of quantum chaos [49,50]. For a perfectly circular structure, the classical dynamics is integrable. For a deformed structure such as one with the stadium shape, there is fully developed chaos in the classical dynamics. We have two main findings. First, in the short-wavelength regime, classical chaos hardly has any effect on the scattering dynamics. In fact, similar behaviors in the scattering characteristics at a quantitative level, such as the skew scattering and backscattering cross sections, arise for the circular and stadium-shaped structures. The diminishing effects of classical chaos on relativistic quantum scattering from a magnetic structure are consistent with previous results on weakened manifestations of chaos in relativistic quantum systems in general [51,52,59-61]. Second, strong skew scattering can arise when the magnetic structure is a skyrmion, regardless of the nature of the classical dynamics. In the short-wavelength regime, the pronounced skew scattering is associated with resonant modes manifested as confined vortices inside the skyrmion structure, which are originated from the sign change in the mass when the Dirac electrons travel from outside to inside the skyrmion structure. A partial-wave analysis for scattering from a circular skyrmion has revealed that a large number of angular momentum channels contribute to the resonant modes. We have also studied the long-wavelength regime, where the geometric details of the magnetic structure are unresolved, so naturally the scattering process is expected to be independent of the nature of the classical dynamics. In this regime, resonant states can still emerge as confined edge states inside the magnetic structure, to which only a single angular momentum channel contributes, leading to highly directional skew scattering.

In the short-wavelength regime, the resonant states manifested as confined vortices inside the skyrmion structure can be exploited for electrically charging the skyrmion structure $[32,33]$, enabling the surface electrons on the TI to drive skyrmion motion with a low current and high thermal efficiency. In the long-wavelength regime, the strong and robust directionality for skew scattering may be exploited for device application based on the anomalous Hall effect.

Regarding experimental realization of a skyrmion structure, we note that there is recent evidence of a magnetic skyrmion at the interface of the ferromagnet/TI $\left(\mathrm{Cr}_{2} \mathrm{Te}_{3} / \mathrm{Bi}_{2} \mathrm{Te}_{3}\right)$ heterostructure [70]. In addition, inhomogeneous Zeeman coupling can be tuned for a ferromagnetic strip with strong out-of-plane magnetic anisotropy [33]. For experimental control of electron scattering over a skyrmion structure, a quantum-dot type of configuration with a skyrmion structure in a finite scattering region as well as with leads and contacts is necessary. The scattering configuration employed in our work is mainly for theoretical convenience with the goal to gain insight into the physics of electron scattering over the skyrmion structure with classical integrable or chaotic dynamics. For this purpose, the geometrical structure of the skyrmion is chosen to be either circular, for which the scattering cross sections can be calculated analytically, or deformed, for which the numerical method of multiple multipoles can be used to calculate the scattering wave function and consequently the resonant states, cross sections, current, and spin distribution. Our results provide useful hints about the scattering of spin$1 / 2$ fermion over a skyrmion structure. If the device size is significantly larger than the electron wavelength, we expect the main results to hold.

A number of open issues are worth studying, such as using spin transfer torque of the electrons to drive the skyrmion motion, exploitation of skyrmion-related switches or oscillators, and scattering from multiple skyrmions that are themselves dynamic with possible phase-locking or antiphase-locking behavior.

\section{ACKNOWLEDGMENTS}

This work was supported by the Pentagon Vannevar Bush Faculty Fellowship program sponsored by the Basic Research 
Office of the Assistant Secretary of Defense for Research and Engineering and funded by the Office of Naval Research through Grant No. N00014-16-1-2828.

\section{APPENDIX: MULTIPLE MULTIPOLE METHOD FOR SCATTERING OF DIRAC ELECTRONS ON TOP OF A TI FROM A MAGNETIC STRUCTURE}

We denote the area outside and inside the skyrmion structure as regions $I$ and $I I$, respectively (see Fig. 13). The wave function in region $I I$ can be written as

$$
\Psi^{I I}(\boldsymbol{r}) \equiv\left(\begin{array}{c}
\psi_{1}^{I} \\
\psi_{2}^{I I}
\end{array}\right)=\sum_{m_{I}} \sum_{l} \frac{C_{l}^{m_{I}}}{\sqrt{2}}\left(\begin{array}{c}
H_{l-1}^{(1)}\left(k_{I I} d_{m_{I}}\right) e^{-i \theta_{m_{I}}} \\
\tau_{I I} H_{l}^{(1)}\left(k_{I I} d_{m_{I}}\right)
\end{array}\right) e^{i l \theta_{m_{I}}},
$$

where

$$
\begin{aligned}
k_{I I} & =\sqrt{E^{2}-\Delta^{2} n_{I I}^{2}} / \hbar v_{F}, \\
\tau_{I I} & =-\hbar v_{F} k_{I I} /\left(E-\Delta n_{I I}\right), \\
d_{m_{I}} & =\left|\boldsymbol{r}-\boldsymbol{r}_{m_{I}}\right|, \\
\theta_{m_{I}} & =\varphi\left(\boldsymbol{r}-\boldsymbol{r}_{m_{I}}\right),
\end{aligned}
$$

where $\phi(v)$ represents the angle of vector $v, C_{l}^{m_{I}}$ are the expansion coefficients, and $H_{l}^{(1)}$ is the Hankel function of the first kind of order $l$. The scattered wave function in region $I$ is $\Psi^{I}(\boldsymbol{r}) \equiv\left(\begin{array}{c}\psi_{1}^{I} \\ \psi_{2}^{I}\end{array}\right)=\sum_{m_{I I}} \sum_{l} \frac{C_{l}^{m_{I I}}}{\sqrt{2}}\left(\begin{array}{c}H_{l-1}^{(1)}\left(k_{I} d_{m_{I I}}\right) e^{-i \theta_{m_{I I}}} \\ \tau_{I} H_{l}^{(1)}\left(k_{I} d_{m_{I I}}\right)\end{array}\right) e^{i l \theta_{m_{I I}}}$,

where

$$
\begin{aligned}
k_{I} & =\sqrt{\left(E-\Delta^{2} n_{I}^{2}\right)} / \hbar v_{F}, \\
\tau_{I} & =-\hbar v_{F} k_{I} /\left(E-\Delta n_{I}\right), \\
d_{m_{I I}} & =\left|\boldsymbol{r}-\boldsymbol{r}_{m_{I I}}\right|, \\
\theta_{m_{I I}} & =\varphi\left(\boldsymbol{r}-\boldsymbol{r}_{m_{I I}}\right),
\end{aligned}
$$

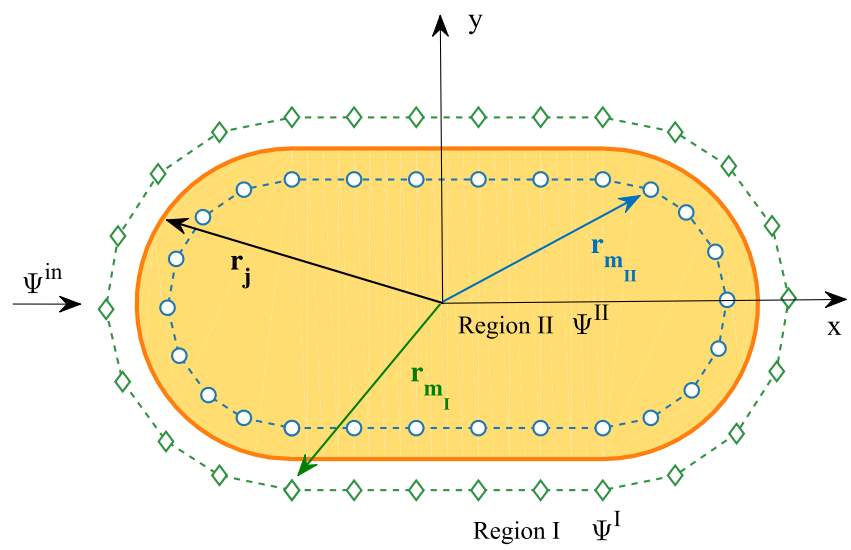

FIG. 13. Schematic illustration of the basics of the MMP method. The placement of poles (fictitious sources) is shown inside and outside a magnetic structure of arbitrary shape. The scattering spinor wave functions inside (outside) the structure are determined by the poles outside (inside) the structure. and $C_{l}^{m_{I I}}$ are the expansion coefficients. The incident plane wave propagating along the direction defined by an angle $\beta$ with the $x$ axis in region $I$ is given by

$$
\Psi^{\text {in }}(\boldsymbol{r}) \equiv\left(\begin{array}{c}
\psi_{1}^{\text {in }} \\
\psi_{2}^{\text {in }}
\end{array}\right)=\frac{1}{\sqrt{2}}\left(\begin{array}{c}
1 \\
-i \tau_{I} e^{i \beta}
\end{array}\right) e^{i\left(k_{x} r \cos \theta+k_{y} r \sin \theta\right)} .
$$

Matching the boundary conditions

$$
\begin{aligned}
& \left.\left(\psi_{1}^{I}+\psi_{1}^{\mathrm{in}}\right)\right|_{r_{j} \in \Gamma}=\left.\psi_{1}^{I I}\right|_{r_{j} \in \Gamma}, \\
& \left.\left(\psi_{2}^{I}+\psi_{2}^{\mathrm{in}}\right)\right|_{r_{j} \in \Gamma}=\left.\psi_{2}^{I I}\right|_{r_{j} \in \Gamma},
\end{aligned}
$$

we get

$$
\begin{aligned}
\sum_{m_{I I}} & \sum_{l} C_{l}^{m_{I I}} \frac{1}{\sqrt{2}} \tau_{I} H_{l}^{(1)}\left(k_{I}\left|\boldsymbol{r}_{j}-\boldsymbol{r}_{m_{I I}}\right|\right) e^{i l \theta_{m_{I I}}} \\
& -\sum_{m_{I}} \sum_{l} C_{l}^{m_{I}} \frac{1}{\sqrt{2}} \tau_{I I} H_{l}^{(1)}\left(k_{I I}\left|\boldsymbol{r}_{j}-\boldsymbol{r}_{m_{I}}\right|\right) e^{i l \theta_{m_{I}}} \\
= & \frac{i}{\sqrt{2}} \tau_{I} e^{i \beta} e^{i \boldsymbol{k}_{\boldsymbol{I}} \boldsymbol{r}}
\end{aligned}
$$

and

$$
\begin{aligned}
\sum_{m_{I I}} & \sum_{l} C_{l}^{m_{I I}} \frac{1}{\sqrt{2}} H_{l-1}^{(1)}\left(k_{I}\left|\boldsymbol{r}_{j}-\boldsymbol{r}_{m_{I I}}\right|\right) e^{i(l-1) \theta_{m_{I I}}} \\
& -\sum_{m_{I}} \sum_{l} C_{l}^{m_{I}} \frac{1}{\sqrt{2}} H_{l-1}^{(1)}\left(k_{I I}\left|\boldsymbol{r}_{j}-\boldsymbol{r}_{m_{I}}\right|\right) e^{i(l-1) \theta_{m_{I}}} \\
= & -\frac{1}{\sqrt{2}} \tau_{I} e^{i \beta} e^{i \boldsymbol{k}_{I} \boldsymbol{r}}
\end{aligned}
$$

which can be cast in a compact form as

$$
\begin{aligned}
& \sum_{m_{I I}} \sum_{l}{ }^{j} A_{l m_{I I}^{I}}^{I} C_{l}^{m_{I I}}-\sum_{m_{I}} \sum_{l}{ }^{j} A_{l m_{I}}^{I I} C_{l}^{m_{I}}=-{ }^{j} \psi_{2}^{\mathrm{in}}, \\
& \sum_{m_{I I}} \sum_{l}{ }^{j} B_{l m_{I I}}^{I} C_{l}^{m_{I I}}-\sum_{m_{I}} \sum_{l}{ }^{j} B_{l m_{I} I}^{I I} C_{l}^{m_{I}}=-{ }^{j} \psi_{1}^{\mathrm{in}},
\end{aligned}
$$

where

$$
\begin{aligned}
{ }^{j} A_{l m_{I I}}^{I} & =\frac{1}{\sqrt{2}} \tau_{I} H_{l}^{(1)}\left(k_{I}\left|\boldsymbol{r}_{j}-\boldsymbol{r}_{m_{I I}}\right|\right) e^{i l \theta_{m_{I I}},} \\
{ }^{j} A_{l m_{I}}^{I I} & =\frac{1}{\sqrt{2}} \tau_{I I} H_{l}^{(1)}\left(k_{I I}\left|\boldsymbol{r}_{j}-\boldsymbol{r}_{m_{I}}\right|\right) e^{i l \theta_{m_{I}}}, \\
{ }^{j} B_{l m_{I I}}^{I} & =\frac{1}{\sqrt{2}} H_{l-1}^{(1)}\left(k_{I}\left|\boldsymbol{r}_{j}-\boldsymbol{r}_{m_{I I}}\right|\right) e^{i(l-1) \theta_{m_{I I}},} \\
{ }^{j} B_{l m_{I}}^{I I} & =\frac{1}{\sqrt{2}} H_{l-1}^{(1)}\left(k_{I I}\left|\boldsymbol{r}_{j}-\boldsymbol{r}_{m_{I}}\right|\right) e^{i(l-1) \theta_{m_{I}}}
\end{aligned}
$$

and

$$
\begin{gathered}
{ }^{j} \psi_{2}^{\text {in }}=-\frac{i}{\sqrt{2}} \tau_{I} e^{i \beta} e^{i \boldsymbol{k}_{I} \boldsymbol{r}_{j}}, \\
{ }^{j} \psi_{1}^{\text {in }}=\frac{1}{\sqrt{2}} e^{i \boldsymbol{k}_{I} \boldsymbol{r}_{j}} .
\end{gathered}
$$

In principle, the set consists of an infinite number of equations with an infinite number of undetermined expansion coefficients $C_{l}^{m_{I I}}$ and $C_{l}^{m_{I}}$. To solve the system numerically, finite truncation is necessary. We set the total number of boundary 
points to be $J$ with $M_{I}$ and $M_{I I}$ poles in regions $I$ and $I I$, respectively, and $l \rightarrow[-L, L]$ for all the multipoles. The process leads to the finite-dimensional matrix equation

$$
M_{2 J \times N} \cdot C_{N \times 1}=-Y_{2 J \times 1},
$$

where $N=(2 L+1) \times\left(M_{I}+M_{I I}\right)=N_{I}+N_{I I}$,

$$
C_{N \times 1}=\left(\begin{array}{c}
C_{-L}^{1_{I I}} \\
\vdots \\
C_{l}^{1_{I I}} \\
C_{l}^{2_{I I}} \\
\vdots \\
C_{l}^{M_{I I}} \\
\vdots \\
C_{L}^{M_{I I}} \\
\hline C_{-L}^{1_{I}} \\
\vdots \\
C_{l}^{1_{I}} \\
C_{l}^{2_{I}} \\
\vdots \\
C_{l}^{M_{I}} \\
\vdots \\
C_{L}^{M_{I}}
\end{array}\right]_{N \times 1} \quad Y_{2 J \times 1}=\left(\begin{array}{c}
{ }^{1} \psi_{2}^{\mathrm{in}} \\
\vdots \\
{ }^{j} \psi_{2}^{\mathrm{in}} \\
\vdots \\
J \psi_{2}^{\mathrm{in}} \\
{ }^{1} \psi_{1}^{\mathrm{in}} \\
\vdots \\
{ }^{j} \psi_{1}^{\mathrm{in}} \\
\vdots \\
J \psi_{1}^{\mathrm{in}}
\end{array}\right)_{2 J \times 1}
$$

and

$$
M_{2 J \times N}=\left(\begin{array}{ll}
\mathbb{A}^{(I)} & -\mathbb{A}^{(I I)} \\
\mathbb{B}^{(I)} & -\mathbb{B}^{(I I)}
\end{array}\right)
$$

with

$$
A^{(\tau)}=\left(\begin{array}{cccccccc}
{ }^{1} A_{-L 1_{\bar{\tau}}}^{(\tau)} & \ldots & { }^{1} A_{l 1_{\bar{\tau}}}^{(\tau)} & { }^{1} A_{l 2_{\bar{\tau}}}^{(\tau)} & \ldots & { }^{1} A_{l M_{\bar{\tau}}}^{(\tau)} & \ldots & { }^{1} A_{L M_{\bar{\tau}}}^{(\tau)} \\
{ }^{2} A_{-L 1_{\bar{\tau}}}^{(\tau)} & \ldots & { }^{2} A_{l 1_{\bar{\tau}}}^{(\tau)} & { }^{2} A_{l 2_{\bar{\tau}}}^{(\tau)} & \ldots & { }^{2} A_{l M_{\bar{\tau}}}^{(\tau)} & \ldots & { }^{2} A_{L M_{\bar{\tau}}}^{(\tau)} \\
\vdots & \ldots & \vdots & \vdots & \ldots & \vdots & \ldots & \vdots \\
{ }^{j} A_{-L 1_{\bar{\tau}}}^{(\tau)} & \ldots & { }^{j} A_{l 1_{\bar{\tau}}}^{(\tau)} & { }^{j} A_{l 2_{\bar{\tau}}}^{(\tau)} & \ldots & { }^{j} A_{l M_{\bar{\tau}}}^{(\tau)} & \ldots & { }^{j} A_{L M_{\bar{\tau}}}^{(\tau)} \\
\vdots & \ldots & \vdots & \vdots & \ldots & \vdots & \ldots & \vdots \\
{ }^{J} A_{-L 1_{\bar{\tau}}}^{(\tau)} & \ldots & { }^{J} A_{l 1_{\bar{\tau}}}^{(\tau)} & { }^{J} A_{l 2_{\bar{\tau}}}^{(\tau)} & \ldots & { }^{J} A_{l M_{\bar{\tau}}}^{(\tau)} & \ldots & { }^{J} A_{L M_{\bar{\tau}}}^{(\tau)}
\end{array}\right)
$$

$$
\mathbb{B}^{(\tau)}=\left(\begin{array}{cccccccc}
{ }^{1} B_{-L 1_{\bar{\tau}}}^{(\tau)} & \ldots & { }^{1} B_{l 1_{\bar{\tau}}}^{(\tau)} & { }^{1} B_{l 2_{\bar{\tau}}}^{(\tau)} & \ldots & { }^{1} B_{l M_{\bar{\tau}}}^{(\tau)} & \ldots & { }^{1} B_{L M_{\bar{\tau}}}^{(\tau)} \\
{ }^{2} B_{-L 1_{\bar{\tau}}}^{(\tau)} & \ldots & { }^{2} B_{l 1_{\bar{\tau}}}^{(\tau)} & { }^{2} B_{l 2_{\bar{\tau}}}^{(\tau)} & \ldots & { }^{2} B_{l M_{\bar{\tau}}}^{(\tau)} & \ldots & { }^{2} B_{L M_{\bar{\tau}}}^{(\tau)} \\
\vdots & \ldots & \vdots & \vdots & \ldots & \vdots & \ldots & \vdots \\
{ }^{j} B_{-L 1_{\bar{\tau}}}^{(\tau)} & \ldots & { }^{j} B_{l 1_{\bar{\tau}}}^{(\tau)} & { }^{j} B_{l 2_{\bar{\tau}}}^{(\tau)} & \ldots & { }^{j} B_{l M_{\bar{\tau}}}^{(\tau)} & \ldots & { }^{j} B_{L M_{\bar{\tau}}}^{(\tau)} \\
\vdots & \ldots & \vdots & \vdots & \ldots & \vdots & \ldots & \vdots \\
{ }^{{ }^{(\tau)}} B_{-L 1_{\bar{\tau}}}^{(\tau)} & \ldots & { }^{J} B_{l 1_{\bar{\tau}}}^{(\tau)} & { }^{J} B_{l 2_{\bar{\tau}}}^{(\tau)} & \ldots & { }^{J} B_{l M_{\bar{\tau}}}^{(\tau)} & \ldots & { }^{J} B_{L M_{\bar{\tau}}}^{(\tau)}
\end{array}\right) .
$$

Here we denote $\tau=I(I I)$ and $\bar{\tau}=I I(I)$ the complement of $\tau$. As the expansions are generally nonorthogonal, more equations are required than the number of unknowns to enable reduction of an overdetermined matrix system with $2 J \gg N$, which can be solved by the pseudoinverse algorithm $\boldsymbol{C}=$ $-\operatorname{pinv}(M) * \boldsymbol{Y}$.
[1] N. Nagaosa and Y. Tokura, Topological properties and dynamics of magnetic skyrmions, Nat. Nanotech. 8, 899 (2013).

[2] A. Fert, V. Cros, and J. Sampaio, Skyrmions on the track, Nat. Nanotech. 8, 152 (2013).

[3] K. Everschor-Sitte, J. Masell, R. M. Reeve, and M. Kläui, Perspective: Magnetic skyrmions-Overview of recent progress in an active research field, J. Appl. Phys. 124, 240901 (2018).

[4] H. Ochoa and Y. Tserkovnyak, Quantum skyrmionics, Int. J. Mod. Phys. B 33, 1930005 (2019).

[5] S. Mühlbauer, B. Binz, F. Jonietz, C. Pfleiderer, A. Rosch, A. Neubauer, R. Georgii, and P. Böni, Skyrmion lattice in a chiral magnet, Science 323, 915 (2009).

[6] X. Yu, Y. Onose, N. Kanazawa, J. Park, J. Han, Y. Matsui, N. Nagaosa, and Y. Tokura, Real-space observation of a two-dimensional skyrmion crystal, Nature (London) 465, 901 (2010).

[7] F. Jonietz, S. Mühlbauer, C. Pfleiderer, A. Neubauer, W. Münzer, A. Bauer, T. Adams, R. Georgii, P. Böni, R. A. Duine et al., Spin transfer torques in $\mathrm{MnSi}$ at ultralow current densities, Science 330, 1648 (2010).

[8] J. Zang, M. Mostovoy, J. H. Han, and N. Nagaosa, Dynamics of Skyrmion Crystals in Metallic Thin Films, Phys. Rev. Lett. 107, 136804 (2011).
[9] X. Yu, N. Kanazawa, W. Zhang, T. Nagai, T. Hara, K. Kimoto, Y. Matsui, Y. Onose, and Y. Tokura, Skyrmion flow near room temperature in an ultralow current density, Nat. Commun. 3, 988 (2012).

[10] S. Tsesses, E. Ostrovsky, K. Cohen, B. Gjonaj, N. Lindner, and G. Bartal, Optical skyrmion lattice in evanescent electromagnetic fields, Science 361, 993 (2018).

[11] G. Yin, Y. Liu, Y. Barlas, J. Zang, and R. K. Lake, Topological spin Hall effect resulting from magnetic skyrmions, Phys. Rev. B 92, 024411 (2015).

[12] K. S. Denisov, I. V. Rozhansky, N. S. Averkiev, and E. Lähderanta, Electron Scattering on a Magnetic Skyrmion in the Nonadiabatic Approximation, Phys. Rev. Lett. 117, 027202 (2016).

[13] K. Denisov, I. Rozhansky, N. Averkiev, and E. Lähderanta, A nontrivial crossover in topological Hall effect regimes, Sci. Rep. 7, 17204 (2017).

[14] P. B. Ndiaye, C. A. Akosa, and A. Manchon, Topological Hall and spin Hall effects in disordered skyrmionic textures, Phys. Rev. B 95, 064426 (2017).

[15] K. S. Denisov, I. V. Rozhansky, M. N. Potkina, I. S. Lobanov, E. Lähderanta, and V. M. Uzdin, Topological Hall effect for electron scattering on nanoscale skyrmions in external magnetic field, Phys. Rev. B 98, 214407 (2018). 
[16] M. Z. Hasan and C. L. Kane, Colloquium: Topological insulators, Rev. Mod. Phys. 82, 3045 (2010).

[17] X.-L. Qi and S.-C. Zhang, Topological insulators and superconductors, Rev. Mod. Phys. 83, 1057 (2011).

[18] Y. Tserkovnyak and D. Loss, Thin-Film Magnetization Dynamics on the Surface of a Topological Insulator, Phys. Rev. Lett. 108, 187201 (2012).

[19] P. Wei, F. Katmis, B. A. Assaf, H. Steinberg, P. Jarillo-Herrero, D. Heiman, and J. S. Moodera, Exchange-Coupling-Induced Symmetry Breaking in Topological Insulators, Phys. Rev. Lett. 110, 186807 (2013).

[20] F. Katmis, V. Lauter, F. S. Nogueira, B. A. Assaf, M. E. Jamer, P. Wei, B. Satpati, J. W. Freeland, I. Eremin, D. Heiman et al., A high-temperature ferromagnetic topological insulating phase by proximity coupling, Nature (London) 533, 513 (2016).

[21] T. Yokoyama, Y. Tanaka, and N. Nagaosa, Anomalous magnetoresistance of a two-dimensional ferromagnet/ferromagnet junction on the surface of a topological insulator, Phys. Rev. B 81, 121401(R) (2010).

[22] Z. Wu, F. M. Peeters, and K. Chang, Electron tunneling through double magnetic barriers on the surface of a topological insulator, Phys. Rev. B 82, 115211 (2010).

[23] G.-L. Wang, H.-Y. Xu, and Y.-C. Lai, Nonlinear dynamics induced anomalous Hall effect in topological insulators, Sci. Rep. 6, 19803 (2016).

[24] G.-L. Wang, H.-Y. Xu, and Y.-C. Lai, Emergence, evolution, and control of multistability in a hybrid topological quantum/classical system, Chaos 28, 033601 (2018).

[25] I. Garate and M. Franz, Inverse Spin-Galvanic Effect in the Interface between a Topological Insulator and a Ferromagnet, Phys. Rev. Lett. 104, 146802 (2010).

[26] T. Yokoyama, J. Zang, and N. Nagaosa, Theoretical study of the dynamics of magnetization on the topological surface, Phys. Rev. B 81, 241410(R) (2010).

[27] T. Yokoyama, Current-induced magnetization reversal on the surface of a topological insulator, Phys. Rev. B 84, 113407 (2011).

[28] Y. G. Semenov, X. Duan, and K. W. Kim, Voltage-driven magnetic bifurcations in nanomagnet-topological insulator heterostructures, Phys. Rev. B 89, 201405(R) (2014).

[29] X. Duan, X.-L. Li, Y. G. Semenov, and K. W. Kim, Nonlinear magnetic dynamics in a nanomagnet-topological insulator heterostructure, Phys. Rev. B 92, 115429 (2015).

[30] P. B. Ndiaye, C. A. Akosa, M. H. Fischer, A. Vaezi, E.-A. Kim, and A. Manchon, Dirac spin-orbit torques and charge pumping at the surface of topological insulators, Phys. Rev. B 96, 014408 (2017).

[31] C.-Z. Wang, H.-Y. Xu, N. D. Rizzo, R. A. Kiehl, and Y.-C. Lai, Phase Locking of a Pair of Ferromagnetic Nano-Oscillators on a Topological Insulator, Phys. Rev. Appl. 10, 064003 (2018).

[32] K. Nomura and N. Nagaosa, Electric charging of magnetic textures on the surface of a topological insulator, Phys. Rev. B 82, 161401(R) (2010).

[33] H. M. Hurst, D. K. Efimkin, J. Zang, and V. Galitski, Charged skyrmions on the surface of a topological insulator, Phys. Rev. B 91, 060401(R) (2015).

[34] Y. Araki and K. Nomura, Skyrmion-induced anomalous Hall conductivity on topological insulator surfaces, Phys. Rev. B 96, 165303 (2017).
[35] X. Zhang, J. Xia, Y. Zhou, D. Wang, X. Liu, W. Zhao, and M. Ezawa, Control and manipulation of a magnetic skyrmionium in nanostructures, Phys. Rev. B 94, 094420 (2016).

[36] O. V. Pylypovskyi, D. Makarov, V. P. Kravchuk, Y. Gaididei, A. Saxena, and D. D. Sheka, Chiral Skyrmion and Skyrmionium States Engineered by the Gradient of Curvature, Phys. Rev. Appl. 10, 064057 (2018).

[37] B. Göbel, A. Schäffer, J. Berakdar, I. Mertig, and S. Parkin, Electrical writing, deleting, reading, and moving of magnetic skyrmioniums in a racetrack device, Sci. Rep. 9, 12119 (2019).

[38] F. S. Nogueira, I. Eremin, F. Katmis, J. S. Moodera, J. van den Brink, and V. P. Kravchuk, Fluctuation-induced Néel and Bloch skyrmions at topological insulator surfaces, Phys. Rev. B 98 , 060401(R) (2018).

[39] M. Garnier, A. Mesaros, and P. Simon, Topological superconductivity with deformable magnetic skyrmions, Commun. Phys. 2, 126 (2019).

[40] S. Rachel, E. Mascot, S. Cocklin, M. Vojta, and D. K. Morr, Quantized charge transport in chiral Majorana edge modes, Phys. Rev. B 96, 205131 (2017).

[41] E. Mascot, S. Cocklin, S. Rachel, and D. K. Morr, Dimensional tuning of Majorana fermions and real space counting of the Chern number, Phys. Rev. B 100, 184510 (2019).

[42] U. Güngördü, S. Sandhoefner, and A. A. Kovalev, Stabilization and control of Majorana bound states with elongated skyrmions, Phys. Rev. B 97, 115136 (2018).

[43] Y. Liu, R. K. Lake, and J. Zang, Shape dependent resonant modes of skyrmions in magnetic nanodisks, J. Magn. Magn. Mater. 455, 9 (2018).

[44] M. V. Berry and R. J. Mondragon, Neutrino billiards: Timereversal symmetry-breaking without magnetic fields, Proc. R. Soc. London Ser. A 412, 53 (1987).

[45] Y.-C. Lai, H.-Y. Xu, L. Huang, and C. Grebogi, Relativistic quantum chaos: An emergent interdisciplinary field, Chaos $\mathbf{2 8}$ 052101 (2018).

[46] L. Huang, H.-Y. Xu, C. Grebogi, and Y.-C. Lai, Relativistic quantum chaos, Phys. Rep. 753, 1 (2018).

[47] A. H. C. Neto and K. Novoselov, Two-dimensional crystals: Beyond graphene, Mater. Exp. 1, 10 (2011).

[48] P. Ajayan, P. Kim, and K. Banerjee, Two-dimensional van der Waals materials, Phys. Today 69(9), 38 (2016).

[49] H.-J. Stöckmann, Quantum Chaos: An Introduction (Cambridge University Press, New York, 2006).

[50] F. Haake, Quantum Signatures of Chaos, 3rd ed., Springer Series in Synergetics Vol. 54 (Springer, Berlin, 2010).

[51] H.-Y. Xu and Y.-C. Lai, Pseudospin-1 wave scattering that defies chaos $Q$-spoiling and Klein tunneling, Phys. Rev. B 99, 235403 (2019).

[52] C.-D. Han, H.-Y. Xu, L. Huang, and Y.-C. Lai, Manifestations of chaos in relativistic quantum systems-A study based on outof-time-order correlator, Phys. Open 1, 100001 (2019).

[53] R. Blümel and U. Smilansky, Classical Irregular Scattering and Its Quantum-Mechanical Implications, Phys. Rev. Lett. 60, 477 (1988).

[54] R. A. Jalabert, H. U. Baranger, and A. D. Stone, Conductance Fluctuations in the Ballistic Regime: A Probe of Quantum Chaos? Phys. Rev. Lett. 65, 2442 (1990).

[55] Y.-C. Lai, R. Blümel, E. Ott, and C. Grebogi, Quantum Manifestations of Chaotic Scattering, Phys. Rev. Lett. 68, 3491 (1992). 
[56] C. M. Marcus, A. J. Rimberg, R. M. Westervelt, P. F. Hopkins, and A. C. Gossard, Conductance Fluctuations and Chaotic Scattering in Ballistic Microstructures, Phys. Rev. Lett. 69, 506 (1992).

[57] R. Ketzmerick, Fractal conductance fluctuations in generic chaotic cavities, Phys. Rev. B 54, 10841 (1996).

[58] R. Yang, L. Huang, Y.-C. Lai, and L. M. Pecora, Modulating quantum transport by transient chaos, Appl. Phys. Lett. 100, 093105 (2012).

[59] R. Yang, L. Huang, Y.-C. Lai, and C. Grebogi, Quantum chaotic scattering in graphene systems, Europhys. Lett. 94, 40004 (2011).

[60] L. Ying, L. Huang, Y.-C. Lai, and C. Grebogi, Conductance fluctuations in graphene systems: The relevance of classical dynamics, Phys. Rev. B 85, 245448 (2012).

[61] H.-Y. Xu, L. Huang, Y.-C. Lai, and C. Grebogi, Superpersistent currents and whispering gallery modes in relativistic quantum chaotic systems, Sci. Rep. 5, 8963 (2015).

[62] H.-Y. Xu, L. Huang, and Y.-C. Lai, A robust relativistic quantum two-level system with edge-dependent currents and spin polarization, Europhys. Lett. 115, 20005 (2016).
[63] A. Larkin and Y. N. Ovchinnikov, Quasiclassical method in the theory of superconductivity, Sov. Phys. JETP 28, 1200 (1969).

[64] C. Schütte and M. Garst, Magnon-skyrmion scattering in chiral magnets, Phys. Rev. B 90, 094423 (2014).

[65] Y. Leviatan and A. Boag, Analysis of electromagnetic scattering from dielectric cylinders using a multifilament current model, IEEE Trans. Antenn. Propag. 35, 1119 (1987).

[66] M. G. Imhof, Multiple multipole expansions for elastic scattering, J. Acoust. Soc. Am. 100, 2969 (1996).

[67] D. I. Kaklamani and H. T. Anastassiu, Aspects of the method of auxiliary sources (MAS) in computational electromagnetics, IEEE Antenn. Propag. Mag. 44, 48 (2002).

[68] E. Moreno, D. Erni, C. Hafner, and R. Vahldieck, Multiple multipole method with automatic multipole setting applied to the simulation of surface plasmons in metallic nanostructures, J. Opt. Soc. Am. A 19, 101 (2002).

[69] G. Tayeb and S. Enoch, Combined fictitious-sourcesscattering-matrix method, J. Opt. Soc. Am. A 21, 1417 (2004).

[70] J. Chen, L. Wang, M. Zhang, L. Zhou, R. Zhang, L. Jin, X. Wang, H. Qin, Y. Qiu, J. Mei et al., Evidence for magnetic skyrmions at the interface of ferromagnet/topological-insulator heterostructures, Nano Lett. 19, 6144 (2019). 